\title{
Fermi-edge singularity in photoluminescence spectra of modulation-doped AlGaAs/InGaAs/GaAs quantum wells
}

\author{
K GOPALAKRISHNA NAIK*, K S R K RAO ${ }^{\dagger}$, T SRINIVASAN ${ }^{\dagger \dagger}$ and \\ R MURALIDHARAN ${ }^{\dagger \dagger}$ \\ Department of Physics, Mangalore University, Mangalagangotri 574 199, India \\ ${ }^{\dagger}$ Department of Physics, Indian Institute of Science, Bangalore 560 012, India \\ ${ }^{\dagger}$ Solid State Physics Laboratory, Lucknow Road, Delhi 110 054, India
}

MS received 11 September 2008; revised 10 March 2011

\begin{abstract}
The photoluminescence study of Fermi-edge singularity (FES) in modulation-doped pseudomorphic $\mathrm{Al}_{x} \mathrm{Ga}_{1-x} \mathrm{As} / \mathrm{In}_{y} \mathrm{Ga}_{1-y} \mathrm{As} / \mathrm{GaAs}$ quantum well $(\mathrm{QW})$ heterostructures is presented. In the above $\mathrm{QW}$ structures the optical transitions between $n=1$ and $n=2$ electronic subband to the $n=1$ heavy hole subband ( $E_{11}$ and $E_{21}$ transitions, respectively) are observed with FES appearing as a lower energy shoulder to the $E_{21}$ transition. The observed FES is attributed to the Fermi wave vector in the first electronic subband under the conditions of population of the second electronic subband. The FES appears at about $10 \mathrm{meV}$ below $E_{21}$ transition around $4.2 \mathrm{~K}$. Initially it gets stronger with increasing temperature and becomes a distinct peak at about $20 \mathrm{~K}$. Further increase in temperature quenches FES and reaches the base line at around $40 \mathrm{~K}$.
\end{abstract}

Keywords. Photoluminescence; Fermi edge singularity; modulation doping.

\section{Introduction}

Modulation-doped quantum wells (QWs) provide nearly ideal systems for the study of many-body effects such as band-gap renormalization, the Fermi-edge singularity (FES), and shake-up processes. The FES is a many-body enhancement of optical spectra that originates from the response of a degenerate two-dimensional electron gas (2DEG) to the photogeneration or annihilation of a positively charged hole. This interaction results in an enhanced oscillator strength for electrons near the Fermi surface recombining with photogenerated hole. Skolnick et al (1987) were the first to report FES in the optical spectra from the two-dimensional semiconductor systems in photoluminescence (PL) spectra from modulationdoped $\mathrm{In}_{y} \mathrm{Ga}_{1-y} \mathrm{As} / \mathrm{InP}$ QWs. Since then FES has been intensively investigated in modulation-doped $\mathrm{Al}_{x} \mathrm{Ga}_{1-x}$ As/GaAs (Brown et al 1997) and $\mathrm{Al}_{x} \mathrm{Ga}_{1-x} \mathrm{As}_{2} / \mathrm{In}_{y} \mathrm{Ga}_{1-y}$ As/GaAs (Chen et al 1992; Xu et al 1996; Kissel et al 2000, 2002) QWs by PL. Existing theoretical models require either hole localization, and/or coupling of electrons at the Fermi level to empty conduction subband, and an abrupt Fermi surface for FES to be observed (Ruckenstein and Schmitt-Rink 1987; Uenoyama and Sham 1989; Chen et al 1990; Hawrylak et al 1990; 1991a, b, 1992; Mueller 1990; Rodriguez and Tejedor 1993, 1996).

\footnotetext{
*Author for correspondence (gopal_mng@yahoo.com)
}

Theoretical investigations show that FES is very sensitive to any broadening mechanism such as the finite hole mass, the life time contribution from the photogenerated hole, and the temperature. However, there are many experimental observations on FES (Brown et al 1997; van der Meulen et al 1999; Kissel et al 2002) that are not fully understood within the present theoretical framework. Kissel et al (2002) observed Fermi edge enhancement from the Fermi level of $n=1$ electronic subband under the condition of $n=2$ electron subband population with increasing excitation power and temperature. Such enhancement with increasing temperature is contrary to the existing theoretical models. Majority of the reported experimental results on FES are concerned with samples of only one electronic subband population. Except for the work of Kissel et al (2002), a few reported results on FES under the condition of second electronic subband population were observed in photoluminescence excitation spectroscopy (PLE) (Skolnick et al 1991; Fisher et al 1993).

In this article, we present PL measurements on modulation-doped pseudomorphic $\mathrm{Al}_{x} \mathrm{Ga}_{1-x} \mathrm{As} / \mathrm{In}_{y} \mathrm{Ga}_{1-y} \mathrm{As} / \mathrm{GaAs}$ heterostructures. We have observed FES in the above structures when both $n=1$ and $n=2$ subbands are populated. FES is around $10 \mathrm{meV}$ below the recombination peak due to $n=2$ electronic subband to $n=1$ heavy-hole subband ( $E_{21}$ transition). Initially FES gets stronger with increasing temperature and becomes distinct at around $20 \mathrm{~K}$. Further increase in temperature quenches FES and reaches the base line at around $40 \mathrm{~K}$. 


\section{Experimental}

The modulation-doped $\mathrm{Al}_{x} \mathrm{Ga}_{1-x} \mathrm{As} / \mathrm{In}_{y} \mathrm{Ga}_{1-y} \mathrm{As} / \mathrm{GaAs} \mathrm{QWs}$ used in this study were grown on semi-insulating GaAs (001) substrates by MBE. In both the samples a buffer structure consisting of $1 \mu \mathrm{m}$ GaAs layer, $1 \mu \mathrm{m}$ low-temperature grown GaAs layer, a 20 period $\mathrm{Al}_{0.24} \mathrm{Ga}_{0.76} \mathrm{As} / \mathrm{GaAs}(50 \AA / 66 \AA)$ superlattice was grown on the semi-insulating GaAs substrates. On the buffer layers of sample 1 the following layer sequence were grown: $500 \AA$ undoped GaAs layer, $135 \AA$ undoped $\mathrm{In}_{0 \cdot 13} \mathrm{Ga}_{0.87} \mathrm{As}$ strained layer, $66 \AA$ undoped $\mathrm{Al}_{0.24} \mathrm{Ga}_{0.76} \mathrm{As}$ spacer layer, a silicon delta doped $\left(4 \times 10^{12} \mathrm{~cm}^{-2}\right)$ layer, a $350 \AA$ silicon doped $\left(5 \times 10^{17} \mathrm{~cm}^{-3}\right) \mathrm{Al}_{0.24} \mathrm{Ga}_{0.76} \mathrm{As}$ layer, and silicon doped $\left(4 \times 10^{18} \mathrm{~cm}^{-3}\right) 100 \AA \mathrm{GaAs}$ cap layer. Sample 2 consists of $300 \AA$ undoped GaAs layer, $120 \AA$ undoped $\operatorname{In}_{0.13} \mathrm{Ga}_{0.87} \mathrm{As}$ strained layer, $40 \AA$ undoped $\mathrm{Al}_{0.24} \mathrm{Ga}_{0.76} \mathrm{As}$ spacer layer, silicon delta doped $(4 \times$ $\left.10^{12} \mathrm{~cm}^{-2}\right)$ layer, $360 \AA$ silicon doped $\left(5 \times 10^{17} \mathrm{~cm}^{-3}\right)$ $\mathrm{Al}_{0.24} \mathrm{Ga}_{0.76} \mathrm{As}$ layer, and finally $100 \AA$ silicon doped $\left(4 \times 10^{18} \mathrm{~cm}^{-2}\right) \mathrm{GaAs}$ as a cap layer. Sample 1 had an electron sheet density of $n_{\mathrm{s}}=1.5 \times 10^{12} \mathrm{~cm}^{-2}$, while sample 2 had $n_{\mathrm{s}}=1.9 \times 10^{12} \mathrm{~cm}^{-2}$ as determined from Hall measurements at $15 \mathrm{~K}$. The PL measurements were obtained using Fourier transform photoluminescence spectrometer. An argon ion laser operating at $514.5 \mathrm{~nm}$ was used as an excitation source.

\section{Results and discussion}

Figures 1(a) and (b) show PL spectra for various excitation powers at $4.2 \mathrm{~K}$ for samples 1 and 2 , respectively. In both the samples PL peaks due to the optical transitions between $n=1$ and $n=2$ electronic subband to the $n=1$ heavy hole subband ( $E_{11}$ and $E_{21}$ transitions, respectively) are observed with FES appearing as a lower energy shoulder to the $E_{21}$ transition. In sample 1, PL peaks due to $E_{11}$ and $E_{21}$ transitions are observed at 1.349 and $1.405 \mathrm{eV}$, respectively. In sample 2 the corresponding PL transitions are observed at 1.354 and $1.415 \mathrm{eV}$. The low temperature PL line shapes in modulation doped quantum wells are accounted for by the electron-hole recombination occurring through direct as well as impurity-assisted (indirect) process (Lyo and Jones 1988). The contribution of the impurity assisted electron-hole recombination leads to broad asymmetric PL spectra from these QWs. In modulation doped quantum wells, the PL transition from indirect processes primarily occurs due to the electron and hole scattering with the ionized dopants in the barrier regions. The observed differences in the PL spectra in samples 1 and 2 are attributed to the difference in widths of the undoped AlGaAs spacer regions, which separates the doped $\mathrm{AlGaAs}$ barrier region and the undoped InGaAs quantum well, as well as the differences in InGaAs QW widths and 2DEG concentrations. The observed rela- tively larger FWHM of $E_{21}$ transition in sample 2 compared with sample 1 is mainly due to the smaller widths of both spacer layers as well as InGaAs quantum well and

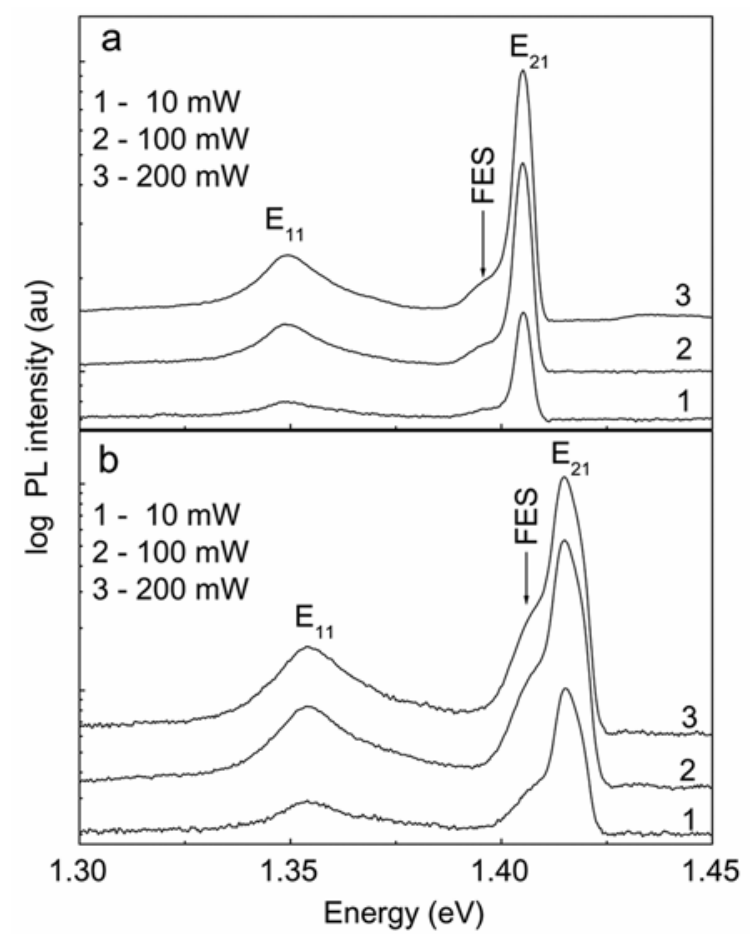

Figure 1. Excitation power dependent evolution of PL spectra in a. sample 1 and b. sample 2 .

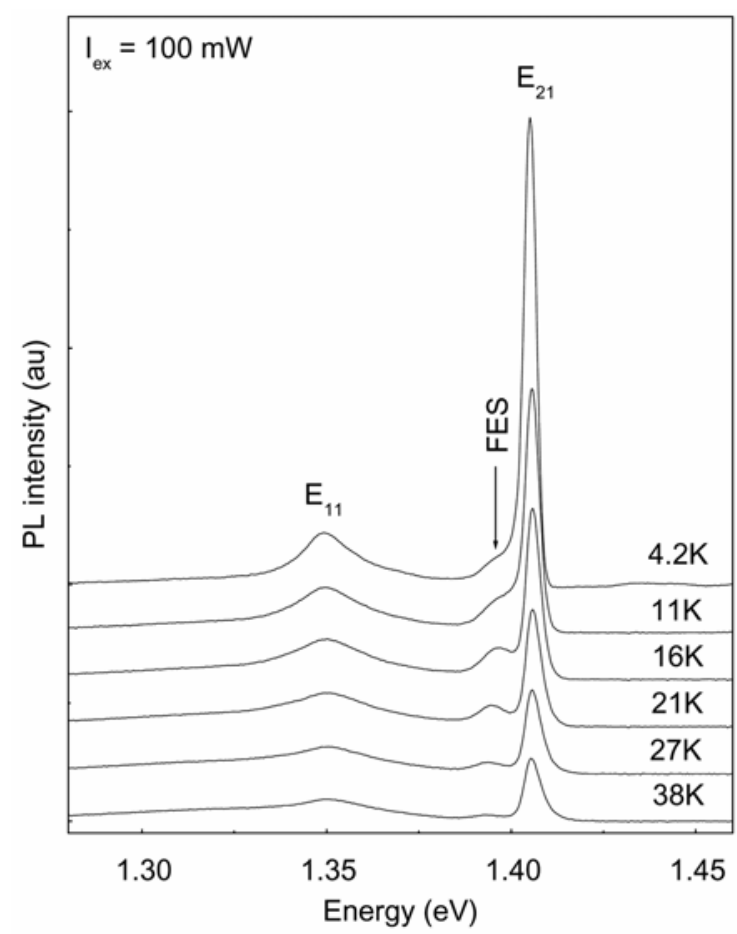

Figure 2. Temperature dependent evolution of PL spectra in sample 1. 
hence increased impurity and disorder assisted electronhole recombination in sample 2 than in sample 1 (Lyo and Jones 1988). The subband structures of these modula-

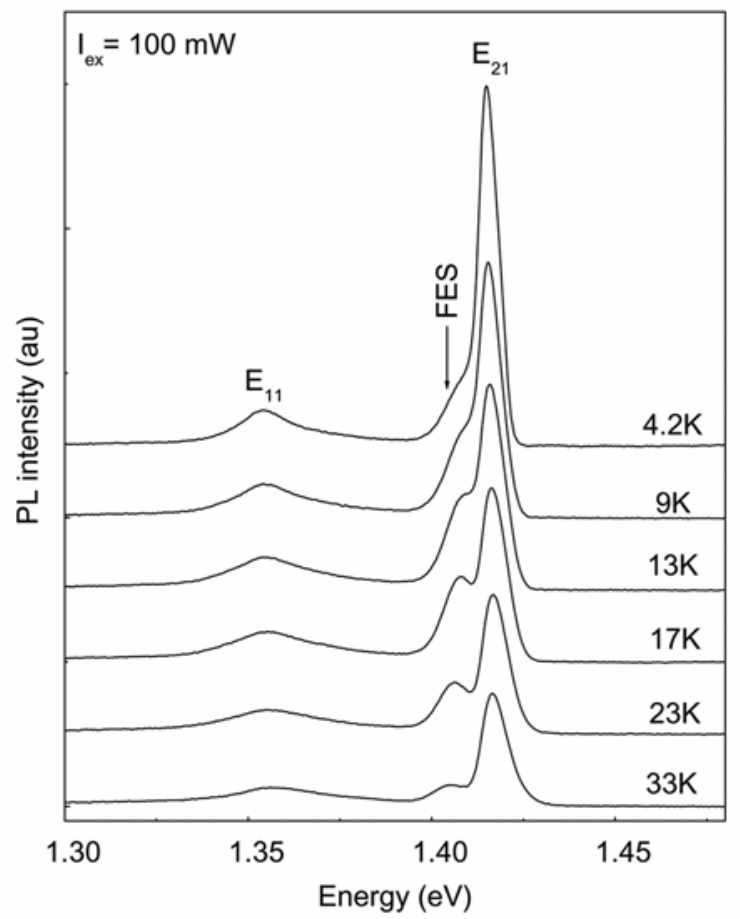

Figure. 3. Temperature dependent evolution of PL spectra in sample 2.

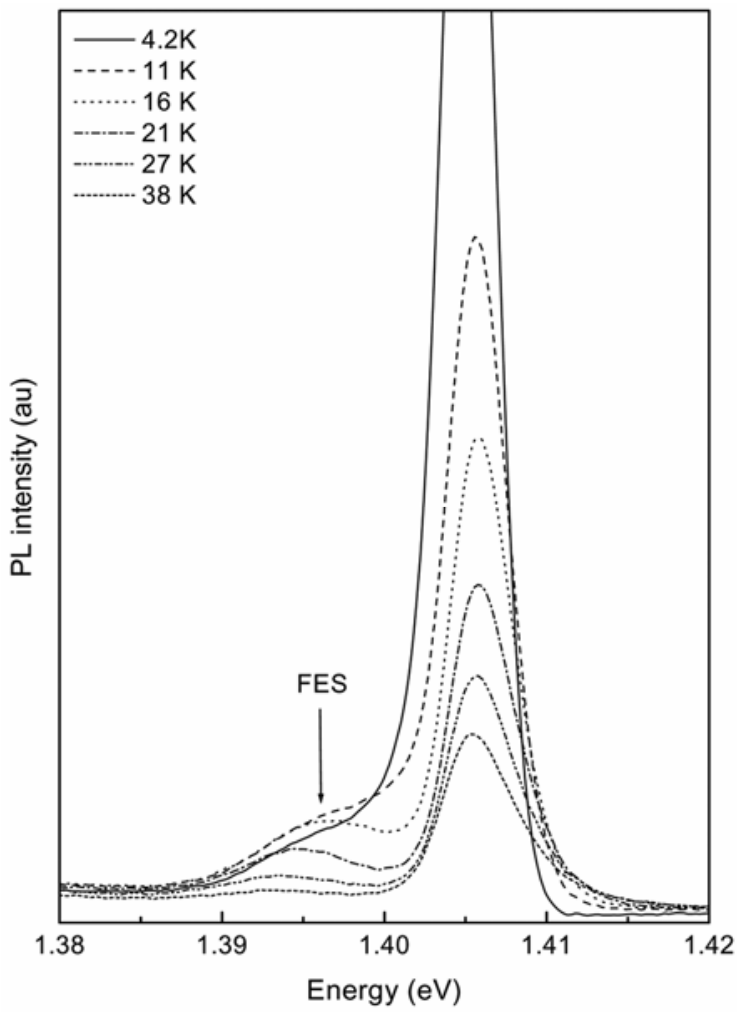

Figure 4. Evolution of FES with temperature in sample 1. tion-doped $\mathrm{Al}_{x} \mathrm{Ga}_{1-x} \mathrm{As} / \mathrm{In}_{y} \mathrm{Ga}_{1-y} \mathrm{As} / \mathrm{GaAs}$ QWs were calculated by self-consistently solving the coupled Schrodinger and Poisson equations using nextnano ${ }^{3}$ device simulation package (http://www.wsi.tu-muenchen.de/nextnano3). The calculations indicate the population of second electronic subband in both the samples as the Fermi level in both the samples is found to be 3 and $6 \mathrm{meV}$, respectively above the bottom of the $n=2$ electronic subband. The temperature dependent PL from samples 1 and 2 is shown in figures 2 and 3, respectively. With increasing temperature, $E_{21}$ transition quenches much faster than $E_{11}$. However, it is observed that FES, which appears as a shoulder ( $10 \mathrm{meV}$ below the $E_{21}$ transition in both samples), grows initially in intensity and evolves into a distinct peak at around $20 \mathrm{~K}$. Further increase in temperature reduces its intensity and disappears around $40 \mathrm{~K}$. An expanded version of the PL spectra showing FES evolution with temperature is shown in Figures 4 and 5 for samples 1 and 2, respectively.

Abbade et al (1996) were the first to report PL spectra similar to that observed by us from modulation doped pseudomorphic $\mathrm{Al}_{x} \mathrm{Ga}_{1-x} \mathrm{As} / \mathrm{In}_{y} \mathrm{Ga}_{1-y} \mathrm{As} / \mathrm{GaAs} \mathrm{QWs}$. They assigned the lower energy peak in the PL to the direct transition involving $n=1$ electronic subband and $n=1$ heavy-hole subband in the $\operatorname{In}_{y} \mathrm{Ga}_{1-y} \mathrm{As} \mathrm{QW}$. The higher energy peak and the additional peak observed at about $7 \mathrm{meV}$ below the higher energy peak in the temperature range of 10-30 K were assigned to the transitions involving the hybrid states in the conduction band and the $n=1$ heavy-hole state. It was predicted that the strong coupling between the $\operatorname{In}_{y} \mathrm{Ga}_{1-y} \mathrm{As} \mathrm{QW}$ and the potential well formed in the $\mathrm{Al}_{x} \mathrm{Ga}_{1-x} \mathrm{As}$ barrier due to planar doping forms these hybrid states in the conduction band. However, the observed temperature variation of the PL intensity of

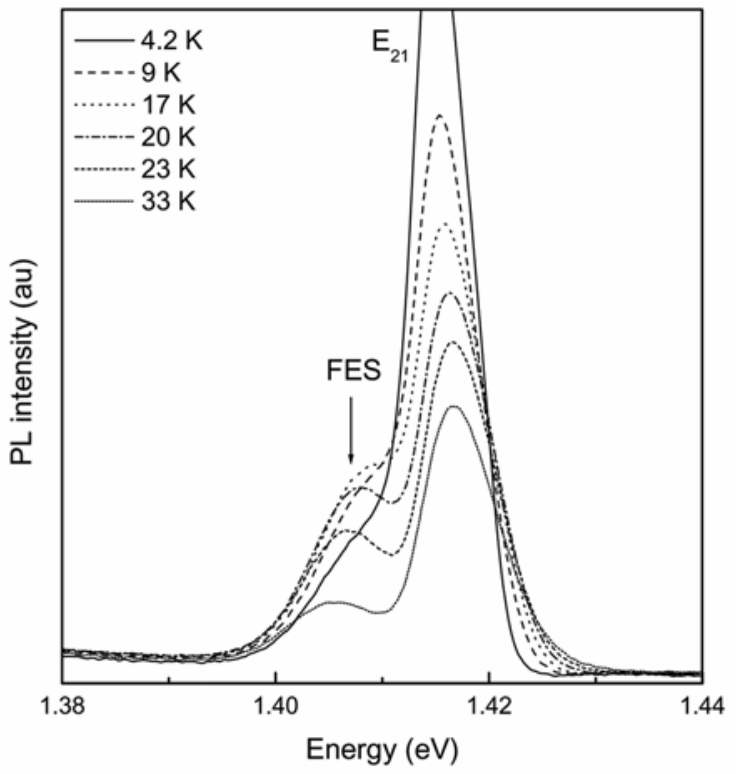

Figure 5. Evolution of FES with temperature in sample 2. 
these peaks contradicts their calculated electron-hole wave function overlaps within the investigated temperature range $(2-50 \mathrm{~K})$. The calculated electron-hole wave function overlap exhibits no substantial variation within the temperature range $(2-50 \mathrm{~K})$ of interest (Abbade et al 1996). Also, it is well known that the higher energy peak in the PL from highly $n$-type modulation doped $\mathrm{Al}_{x} \mathrm{Ga}_{1-x} \mathrm{As} / \mathrm{In}_{y} \mathrm{Ga}_{1-y} \mathrm{As} / \mathrm{GaAs} \mathrm{QWs}$ is attributed to $E_{21}$ transition (Chen et al 1990, 1992; Skolnick et al 1991; Fisher et al 1993). On similar arguments, Kissel et al (2002) ruled out the assignment of the above mentioned peaks to the conduction band hybrid states.

Rodriguez and Tejedor (1996) predicted the appearance of two Fermi-edge singularities arising from the first and second Fermi wave vectors in each conduction subband when the second conduction subband also populated. For the case involving one hole $\left(h h_{1}\right)$ and two conduction ( $e_{1}$ and $e_{2}$ ) subbands it has been shown by Rodriguez and Tejedor that the main contribution to the optical spectrum in the ladder approximation will arise from the $G_{00 ; 10}+G_{01 ; 00}$ terms in the Bethe-Salpeter equations for interacting electron-hole Greeen's functions $G_{i j ; k l}$. In this case the off-diagonal terms, $G_{00 ; 01}$ represents the mixing of the $h h_{1}-e_{1}$ and $h h_{1}-e_{2}$ transitions and it will be responsible for the enhancement of FES in luminescence spectra. Therefore, in the three band systems, two singularities should appear arising from the first and second Fermi wave vectors in each electronic subband.

In our samples only a slight filling of the second electronic subband in the InGaAs QW is expected. Therefore, in the PL spectrum, FES arising from the Fermi wave vector corresponding to the slightly filled $n=2$ electronic subband will occur close to the $E_{21}$ transition energy. However, FES from the Fermi wave vector corresponding to the $n=1$ electronic subband will occur at an energy shifted below the $E_{21}$ transition by a value corresponding to the heavy hole localization energy (Kissel et al 2002). The momentum conservation requirements needs localized heavy holes for the observation of FES from the Fermi wave vector corresponding to the $n=1$ electronic subband (Kissel et al 2002). The localization spreads the hole wave function sufficiently in $k$-space to allow these transitions otherwise forbidden due to momentum conservation. The hole localization in these $\mathrm{Al}_{x} \mathrm{Ga}_{1-x} \mathrm{As} /$ $\mathrm{In}_{y} \mathrm{Ga}_{1-y} \mathrm{As} / \mathrm{GaAs} \mathrm{QWs}$ may be due to the disorder arising from the alloy fluctuations in $\mathrm{In}_{y} \mathrm{Ga}_{1-y}$ As QWs (Skolnick et al 1987).

\section{Conclusions}

In conclusion, we have carried out PL investigations on modulation-doped $\mathrm{Al}_{x} \mathrm{Ga}_{1-x} \mathrm{As} / \mathrm{In}_{y} \mathrm{Ga}_{1-y} \mathrm{As} / \mathrm{GaAs} \mathrm{QW}$ heterostructures. It is interesting to observe FES, which is a many body phenomenon, as a lower energy shoulder to the $E_{21}$ (10 meV below) along with the regular $E_{11}$ and $E_{21}$ transitions related to electronic ( $n=1$ and 2 ) and hole $(n=1)$ subbands. The signature of FES is observed only at low temperatures. With increasing temperature, FES goes through a maximum at about $20 \mathrm{~K}$ and completely gets annealed off around $40 \mathrm{~K}$. Though, such anomalous temperature dependence of FES is not clearly understood within the existing theoretical models, its appearance as a low energy shoulder to $E_{21}$ is explained in the frame work of hole-localization phenomenon.

\section{References}

Abbade M L F, Iikawa F, Brum J A, Troster Th., Bemussi A A, Pereira R G and Borghs G 1996 J. Appl. Phys. 801925

Brown S A, Young J F, Wasilewski Z and Coleridge P T 1997 Phys. Rev. B56 3937

Chen W, Fritze M, Nurmikko A V, Ackley D, Colvard C and Lee H 1990 Phys. Rev. Lett. 642434

Chen W, Fritze M, Walecki W, Nurmikko A V, Ackley D, Hong J M and Chang L L 1992 Phys. Rev. B45 8464

Fisher T A, Simmonds P E, Skolnick M S, Martin A D and Smith R S 1993 Phys. Rev. B48 14253

Hawrylak P 1990 Phys. Rev. B42 8986

Hawrylak P 1991a Phys. Rev. B44 3821

Hawrylak P 1991b Phys. Rev. B44 6262

Hawrylak P 1992 Phys. Rev. B45 4237

Kissel H et al 2000 Phys. Rev. B61 8359

Kissel H et al 2002 Phys. Rev. B65 235320

Lyo S K and Jones E D 1988 Phys. Rev. B38 4113

Mueller J F 1990 Phys. Rev. B42 189

Rodriguez F J and Tejedor C 1993 Phys. Rev. B47 1506

Rodriguez F J and Tejedor C 1996 J. Phys: Condens. Matter 8 1713

Ruckenstein A E and Schmitt-Rink S 1987 Phys. Rev. B35 7551

Skolnick M S, Rorison J M, Nash K J, Mowbray D J, Tapster P R, Bass S J and Pitt A D 1987 Phys. Rev. Lett. 582130

Skolnick M S et al 1991 Phys. Rev. B43 7354

Uenoyama T and Sham L J 1989 Phys. Rev. B39 11044

van der Meulen H P, Santa-Olalla I, Rubio J, Calleja J M, Friedland K J, Hey R and Ploog K 1999 Phys. Rev. B60 4897

Xu S J, Chua S J, Tang X H and Zhang X H 1996 Phys. Rev. B54 17701 